\title{
Mechanical analysis and modeling of collision in flight process for table tennis
}

\author{
Zixin $\mathrm{Zhu}^{1}$, Fu Hui ${ }^{1}$ \\ ${ }^{1}$ Huazhong Agricultural University, Wuhan Hubei, China \\ ahubzzx@163.com
}

Keywords: mechanical analysis, force analysis, modeling, flight, table tennis.

\begin{abstract}
Collision is of great importance in many modern sports, especially in table tennis. Research on collision should first pays attention to modeling the flight process. The paper analyzes the kinematic mechanics of collision in flight process for table tennis. It first discusses the great importance of modeling flight process for table tennis. Then, by means of the motion analysis, the force situation of the tennis ball in flight process is found out. On the basis of this, three basic assumptions are presented and the force analysis of flight process in three-dimensional coordinate system is conducted. At last, the model of collision in flight process is established by means of mathematical deduce. And the changing rules of velocity and distance are discovered.
\end{abstract}

\section{Introduction}

Collision is an important sport skill in many modern sports, just like table tennis, billiards, etc. Especially for table tennis, mastering good collision skills is a key factor to make success in table tennis. Research on collision skill is of great significance for table tennis [1]. For the collision of tennis ball with table, it firstly needs a period of time of flight in space. Therefore, analysis of flight process in space is a basis to collision.

Thus, in contrast with thorough macroscopic theories and training methods, the theory of mechanical analysis and modeling for collision in flight process appears more weakness. In addition, mechanical analysis and modeling can not only promote the microscopic theory and represent the flight and collision law of table tennis comprehensively and quantitatively, but also the modeling method and modeling process are particularly beneficial for other sport events and disciplines. Hence, mechanical analysis and modeling of collision in flight process for table tennis is taken as the research object in the paper. The primary aim of the research in the paper attempts to present a theoretical model for table tennis by means of kinematics mechanics. The structure of the paper is as follows: the paper begins with the introduction of analyzing the significance of flight process for table tennis; then, it puts forward the assumptions and conducts a force analysis for table tennis in flight process in section 1; the model is established in section 2 to present the moving velocity and distance in flight process for table tennis. The last section sums up the research conclusions.

\section{Basic assumptions and force analysis of flight process for table tennis}

The collision of tennis ball with table differs from the collision between centroid and surface. Furthermore, the whole collision process is not an ideal state. Hence, a more general collision model should be constructed to conduct an effective analysis between tennis ball and tennis table. Before research on the collision, we must study the flight process and the construction of the motion model. So some basic assumptions are first introduced and the force analysis is conducted for table tennis in order to display the collision mechanics [2].

Prediction of the attacking position, attacking time and attacking speed in attacking point primarily depends on the flight model of tennis ball. But the whole motion process of tennis ball in flight is very complex. Seen from the force analysis, there are many forces acting on the tennis ball in the collision, just as force of gravity, air friction, aerostatic buoyancy, and so on. Especially for the aerostatic buoyancy, there are three forms of aerostatic buoyancy in theory. Production of aerostatic buoyancy is due to fluid's characteristics of easiness to flow, viscosity and 
compressibility. These characteristics make the tennis ball moving in the air suffer from the forces of viscous resistance, pressure drag and wave-making resistance.

Generally speaking, wave-making resistance is more applicable to reveal the force situation of ship sailing in the water; viscous resistance results from the interaction between the object and the fluid in the surface of the object; pressure drag roots in the pressure difference behind and before the object. Due to the density in liquid is larger than in gas, the influence of pressure difference acting on the object in liquid is more apparent. For the object moving in the gas, the force situation is frequently related with the moving speed. In a general way, the object with a small moving speed mainly suffers from viscous resistance. On the contrary, an object with a fast moving speed is primarily subjected to pressure drag resistance. For a tennis ball moving in the air, it is a real possibility that the speed frequently ranges from motionless to $7 \mathrm{~m} / \mathrm{s}$. Therefore, the force of wave-making resistance can be omitted for tennis ball moving in the air. In other words, the forces of viscous resistance and pressure drag should be considered.

However, if the nonlinear pressure drag force is introduced, the model will become surprisingly complicated. Even the constructed model will become unstable and the theory analysis will not be conducted. Therefore, the pressure drag force is also ignored and the linear viscous resistance force is only retained.

Now, three assumptions of great significance in the paper are given as follows according to the above analysis:

(a) The rotation of tennis ball moving in the space is neglected. That is to say, the forces in $x, y, z$ three directions are mutual independence.

(b) The forces of pressure drag and wave-making resistance are omitted. Therefore, the tennis ball suffers from the forces of gravity and viscous resistance. Apparently, the viscous resistance force is direct proportion to the moving speed of tennis ball.

(c) The collision of tennis ball with tennis table is elastic, which is related with the incoming motion state.

In accordance with the assumptions proposed above, the force analysis of tennis ball moving in the space is display in Figure 1 as the following.

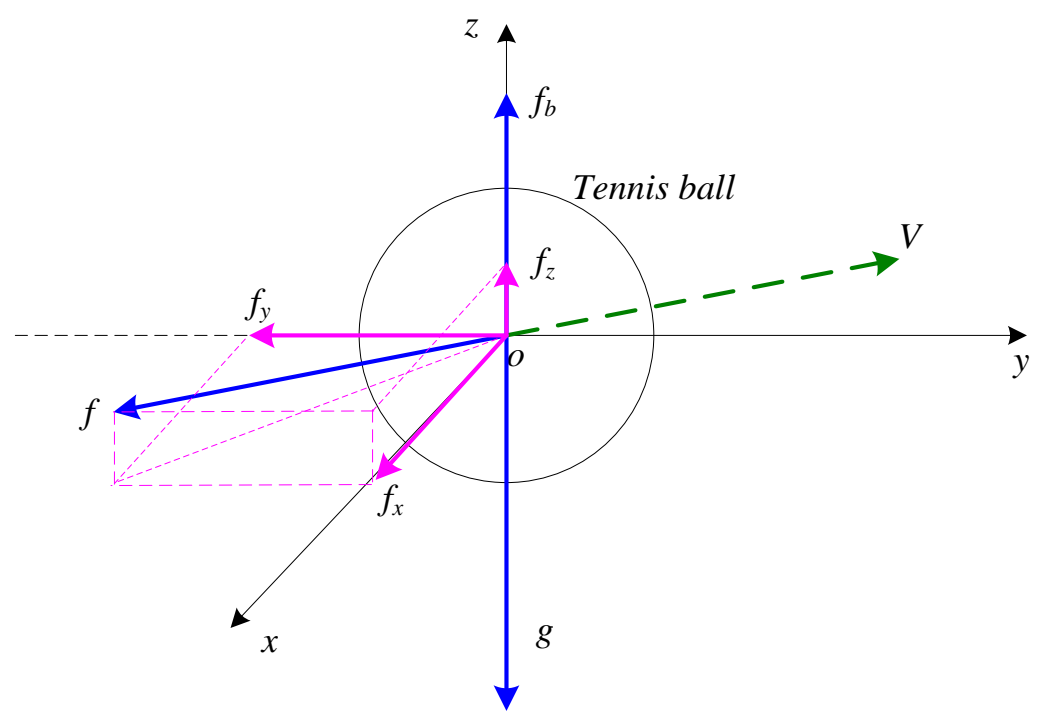

Figure 1. Force analysis of tennis ball moving in the space.

In Figure $1, f, f_{b}$ and $g$ represent the forces of air friction, aerostatic buoyancy and gravity, respectively, where the air friction force points out the viscous resistance force, which can be calculated as bellow:

$$
f=\eta \Delta S\left(\frac{d V}{d y}\right),
$$


where $\eta$ is the viscosity coefficient of the fluid, $\Delta S$ is the sectional area of tennis ball, $\frac{d V}{d y}$ indicates the air velocity gradient. For a spherical tennis ball moving slowly in the air, the viscous resistance force $f$ can be written as follows:

$$
f=6 \pi \eta r V \text {, }
$$

where $r$ is the radius of tennis ball.

In addition, $f_{x}, f_{y}$ and $f_{z}$ in Figure 1 indicate the projection forces in the directions of $x, y, z$ respectively.

\section{Modeling of collision in flight for table tennis}

According to the force analysis in Figure 1 and assumptions put forward in above section, we can get the projection forces $f_{x}, f_{y}$ and $f_{z}$ in Figure 1 as below:

$$
\left\{\begin{array}{l}
F_{x}=f_{x}=-k V_{x} \\
F_{y}=f_{y}=-k V_{y} \\
F_{z}=\left(f_{b}-m g\right)+f_{z}=\left(f_{b}-m g\right)-k V_{z}
\end{array},\right.
$$

where $m$ is the mass of tennis ball, $k$ is the coefficient.

In accordance with the projection forces, the projection accelerated velocities in $x, y$ and $z$ directions can be shown as follows:

$$
\left\{\begin{array}{l}
a_{x}=\frac{F_{x}}{m}=\frac{f_{x}}{m}=-\frac{k}{m} V_{x} \\
a_{y}=\frac{F_{y}}{m}=\frac{f_{y}}{m}=-\frac{k}{m} V_{y} \\
a_{z}=\frac{F_{z}}{m}=\frac{\left(f_{b}-m g\right)+f_{z}}{m}=-\frac{k}{m} V_{z}+\left(\frac{f_{b}}{m}-g\right)
\end{array} .\right.
$$

Hence, the kinematical equation of tennis ball can be shown as the following:

$$
\left\{\begin{array}{l}
V_{x}(t)=V_{x 0}+\int_{0}^{t} a_{x}(\tau) d \tau=V_{x 0}-\int_{0}^{t} \frac{k}{m} V_{x}(\tau) d \tau \\
V_{y}(t)=V_{y 0}+\int_{0}^{t} a_{y}(\tau) d \tau=V_{y 0}-\int_{0}^{t} \frac{k}{m} V_{y}(\tau) d \tau \\
V_{z}(t)=V_{z 0}+\int_{0}^{t} a_{z}(\tau) d \tau=V_{z 0}+\int_{0}^{t}\left(-\frac{k}{m} V_{z}(\tau)+\frac{f_{b}}{m}-g\right) d \tau=V_{z 0}+\left(\frac{f_{b}}{m}-g\right) t-\int_{0}^{t} \frac{k}{m} V_{z}(\tau) d \tau
\end{array},\right.
$$

let $c=\frac{k}{m}$, the calculation formulas of the moving velocity in $x, y$ and $z$ directions can be obtained as the following:

$$
\left\{\begin{array}{l}
V_{x}(t)=V_{x 0} e^{-c t} \\
V_{y}(t)=V_{y 0} e^{-c t} \\
V_{z}(t)=\left(V_{z 0}+\left(\frac{f_{b}}{m}-g\right)\right) e^{-c t}-\left(\frac{f_{b}}{m c}-\frac{g}{c}\right)
\end{array} .\right.
$$

From the velocity formula (8), we can see the velocities in $x, y$ and $z$ directions of the tennis ball in flight process varies with time of exponential rate.

Due to the relationship between the distance and velocity can be written as follows: 


$$
S=\int_{0}^{t} V(\tau) d \tau
$$

Therefore, the moving distance of tennis ball in $\mathrm{x}, \mathrm{y}$ and $\mathrm{z}$ directions can be calculated as the following:

$$
\left\{\begin{array}{l}
S_{x}(t)=\int_{0}^{t} V_{x}(\tau) d \tau=\frac{V_{x 0}}{c}\left(1-e^{-c t}\right) \\
S_{y}(t)=\int_{0}^{t} V_{y}(\tau) d \tau=\frac{V_{y 0}}{c}\left(1-e^{-c t}\right) \\
S_{z}(t)=\int_{0}^{t} V_{z}(\tau) d \tau=\frac{V_{z 0}+\left(\frac{f_{b}}{m}-g\right)}{c}\left(1-e^{-c t}\right)-\left(\frac{f_{b}}{m c}-\frac{g}{c}\right) t
\end{array} .\right.
$$

If the air resistance force is omitted in the analysis, that is to say, the value of $c$ is very small, we can simplify the distance in $x$ direction and the result can be shown as the following:

$$
\lim _{c \rightarrow 0} S_{x}(t)=\lim _{c \rightarrow 0} \frac{V_{x 0}}{c}\left(1-e^{-c t}\right)=V_{x 0} t .
$$

Similarly,

$$
\begin{aligned}
& \lim _{c \rightarrow 0} S_{x y}(t)=\lim _{c \rightarrow 0} \frac{V_{y 0}}{c}\left(1-e^{-c t}\right)=V_{y 0} t . \\
& \lim _{c \rightarrow 0} S_{x y}(t)=\lim _{c \rightarrow 0}\left(\frac{V_{z 0}+\left(\frac{f_{b}}{m}-g\right)}{c}\left(1-e^{-c t}\right)-\left(\frac{f_{b}}{m c}-\frac{g}{c}\right) t\right)=\lim _{c \rightarrow 0}\left(\frac{V_{z 0}+\frac{g}{c}}{c}\left(1-e^{-c t}\right)-\frac{g}{c} t\right) \\
& =\lim _{c \rightarrow 0}\left(\frac{V_{z 0}+\frac{g}{c}}{c}\left(1-\left(1-c t+\frac{(c t)^{2}}{2}\right)\right)-\frac{g}{c} t\right)=\lim _{c \rightarrow 0}\left(\left(V_{z 0}+\frac{g}{c}\right)\left(c t-\frac{c t^{2}}{2}\right)-\frac{g}{c} t\right)=V_{z 0} t-\frac{g t^{2}}{2}
\end{aligned}
$$

The moving distance in $x$, and $y$ directions of the tennis ball in flight process both increase linearly with time, but the moving distance in $z$ direction is more complex, which is .

\section{Conclusions}

This paper analyzes the kinematic mechanics and modeling the flight process for table tennis and the conclusions are summarized as the following:

(a) When the tennis ball flies in the space, there are many forces acting on the tennis ball, which primarily are the forces of air viscous resistance gravity, and aerostatic buoyancy.

(b) According to the established coordinate system, the moving velocities of tennis ball projected into $x, y$ and $z$ directions change with time in the form of exponentially.

(c) The moving distances of tennis ball projected into $x, y$ and $z$ directions differ from the velocities, which increase linearly with time in $x$ and $y$ directions and more complicated in $z$ direction.

\section{References}

[1] Sørensen V., Ingvaldsen R. P., Whiting H. T., in Biological Cybernetics, Vol. 85, 2001, p. 27.

[2] Hughes M. D., Bartlett R. M., in: Journal of sports sciences, Vol. 20, 2002, p. 739. 